\title{
Estimates for Multilinear Commutators of Generalized Fractional Integral Operators on Weighted Morrey Spaces
}

\author{
Sha He, ${ }^{1}$ Taotao Zheng, ${ }^{2}$ and Xiangxing Tao ${ }^{3}$ \\ ${ }^{1}$ School of Mathematical Sciences, Beijing Normal University, and Laboratory of Mathematics and Complex Systems, \\ Ministry of Education, Beijing 100875, China \\ ${ }^{2}$ Department of Mathematics, Zhejiang University, Hangzhou, Zhejiang 310027, China \\ ${ }^{3}$ Department of Mathematics, Zhejiang University of Science and Technology, Hangzhou, Zhejiang 310023, China
}

Correspondence should be addressed to Xiangxing Tao; xxtao@zust.edu.cn

Received 28 July 2014; Accepted 29 October 2014

Academic Editor: Nelson José Merentes Díaz

Copyright $\odot 2015$ Sha He et al. This is an open access article distributed under the Creative Commons Attribution License, which permits unrestricted use, distribution, and reproduction in any medium, provided the original work is properly cited.

Let $L$ be the infinitesimal generator of an analytic semigroup on $L^{2}\left(\mathbb{R}^{n}\right)$ with Gaussian kernel bounds, and let $L^{-\alpha / 2}$ be the fractional integrals of $L$ for $0<\alpha<n$. Assume that $\vec{b}=\left(b_{1}, b_{2}, \ldots, b_{m}\right)$ is a finite family of locally integrable functions; then the multilinear commutators generated by $\vec{b}$ and $L^{-\alpha / 2}$ are defined by $L_{\vec{b}}^{-\alpha / 2} f=\left[b_{m}, \ldots,\left[b_{2},\left[b_{1}, L^{-\alpha / 2}\right]\right], \ldots\right] f$. Assume that $b_{j}$ belongs to weighted BMO space, $j=1,2, \ldots, m$; the authors obtain the boundedness of $L_{\vec{b}}^{-\alpha / 2}$ on weighted Morrey spaces. As a special case, when $L=-\Delta$ is the Laplacian operator, the authors also obtain the boundedness of the multilinear fractional commutator $I_{\alpha}^{\vec{b}}$ on weighted Morrey spaces. The main results in this paper are substantial improvements and extensions of some known results.

\section{Introduction and Main Results}

Assume that $L$ is a linear operator on $L^{2}\left(\mathbb{R}^{n}\right)$, which generates an analytic semigroup $e^{-t L}$ with a kernel $p_{t}(x, y)$ satisfying a Gaussian upper bound; that is,

$$
\left|p_{t}(x, y)\right| \leq \frac{C}{t^{n / 2}} e^{-c\left(|x-y|^{2} / t\right)}
$$

for $x, y \in \mathbb{R}^{n}$ and all $t>0$.

The property (1) is satisfied by a large amount of differential operators. One can see [1] for details and examples.

For $0<\alpha<n$, the fractional integral $L^{-\alpha / 2}$ generated by the operator $L$ is defined by

$$
L^{-\alpha / 2} f(x)=\frac{1}{\Gamma(\alpha / 2)} \int_{0}^{\infty} e^{-t L}(f) \frac{d t}{t^{-\alpha / 2+1}}(x) .
$$

Let $\vec{b}=\left(b_{1}, b_{2}, \ldots, b_{m}\right)$ be a finite family of locally integrable functions; then the multilinear commutators generated by $L^{-\alpha / 2}$ and $\vec{b}$ are defined by

$$
L_{\vec{b}}^{-\alpha / 2} f=\left[b_{m}, \ldots,\left[b_{2},\left[b_{1}, L^{-\alpha / 2}\right]\right], \ldots\right] f
$$

where $m \in \mathbb{Z}^{+}$.

Note that if $L=-\Delta$, which is Laplacian on $\mathbb{R}^{n}$, then $L^{-\alpha / 2}$ is the classical fractional integral $I_{\alpha}$ :

$$
I_{\alpha} f(x)=\frac{\Gamma((n-\alpha) / 2)}{\pi^{n / 2} 2^{\alpha} \Gamma(\alpha / 2)} \int_{\mathbb{R}^{n}} \frac{f(y)}{|x-y|^{n-\alpha}} d y,
$$

while $L_{\vec{b}}^{-\alpha / 2}$ is the iterated commutator generated by $\vec{b}$ and $I_{\alpha}$ :

$$
I_{\alpha}^{\vec{b}} f=\left[b_{m}, \ldots,\left[b_{2},\left[b_{1}, I_{\alpha}\right]\right], \ldots\right] f
$$

where $m \in \mathbb{Z}^{+}$.

When $m=1$, it is easy to see that $L_{\vec{b}}^{-\alpha / 2} f=\left[b, L^{-\alpha / 2}\right] f$ is the commutator generated by $L^{-\alpha / 2}$ and $b$, and when $b_{1}=$ $b_{2}=\cdots=b_{m}, L_{\vec{b}}^{-\alpha / 2}$ is the higher commutator.

As we all know, if $b \in \mathrm{BMO}$, the commutator of fractional integral operator $\left[b, I_{\alpha}\right]$ is bounded from $L^{p}\left(\mathbb{R}^{n}\right)$ to $L^{q}\left(\mathbb{R}^{n}\right)$, where $1<p<n / \alpha$ and $1 / q=1 / p-$ 
$\alpha / n$ (see [2]). In 2004, Duong and Yan [1] generalized the above classical result and obtained the $\left(L^{p}, L^{q}\right)$ boundedness of the commutator $\left[b, L^{-\alpha / 2}\right]$ under the same conditions. Simultaneously, the theory on multilinear integral operators and multilinear commutators has attracted much attention as a rapid developing field in harmonic analysis. Mo and $\mathrm{Lu}$ [3] studied the $\left(L^{p}, L^{q}\right)$ boundedness of the multilinear commutators $L_{\vec{b}}^{-\alpha / 2}$, where $\vec{b}=\left(b_{1}, \ldots, b_{m}\right), b_{j} \in \mathrm{BMO}$, and $j=1,2, \ldots, m$.

On the other hand, Muckenhoupt and Wheeden [4] gave some definitions of weighted bounded mean oscillation and obtained some equivalent conditions for them.

Definition 1 (see [4]). Let $1 \leq p<\infty$ and $w$ be locally integral in $\mathbb{R}^{n}$ and let $w \geq 0$. A locally integrable function $b$ is said to be in $\mathrm{BMO}_{p}(w)$ if

$$
\begin{aligned}
\|b\|_{\mathrm{BMO}_{p}(w)} & =\sup _{\mathrm{Q}}\left(\frac{1}{w(Q)} \int_{\mathrm{Q}}\left|b(x)-b_{\mathrm{Q}}\right|^{p} w(x)^{1-p} d x\right)^{1 / p} \\
& \leq C
\end{aligned}
$$

where $b_{\mathrm{Q}}=(1 /|\mathrm{Q}|) \int_{\mathrm{Q}} b(y) d y$ and the supremum is taken over all balls $Q \in \mathbb{R}^{n}$.

We may note that other weighted definitions for the bounded mean oscillation also have been given by Muckenhoupt and Wheeden in [4].

Definition 2 (see [4]). Let $w$ be locally integral in $\mathbb{R}^{n}$ and $w \geq$ 0 . A locally integrable function $b$ is said to be in $\operatorname{BMO}(w)$ if the norm of $\operatorname{BMO}(w):\|\cdot\|_{*, w}$ satisfies

$$
\|b\|_{*, w}=\sup _{\mathrm{Q}} \frac{1}{w(Q)} \int_{\mathrm{Q}}\left|b(x)-b_{\mathrm{Q}, w}\right| w(x) d x \leq C,
$$

where $b_{\mathrm{Q}, w}=(1 / w(Q)) \int_{Q} b(z) w(z) d z$ and the supremum is taken over all balls $Q \in \mathbb{R}^{n}$.

The above two definitions cannot contain each other; throughout this paper, we will make some investigations on the basis of Definition 2.

Recently, Wang [5] obtained some estimates for the commutator $\left[b, I_{\alpha}\right]$ on weighted Morrey space (see Definitions 3 and 4$)$, where $b \in \mathrm{BMO}_{1}(w)$. Furthermore, Wang and Si [6] obtained the necessary and sufficient conditions for the boundedness of $\left[b, L^{-\alpha / 2}\right]$ on weighted Morrey spaces when $b \in \mathrm{BMO}_{1}(w)$.

Motivated by $[1,3,5,6]$, it is natural to raise the following question: how to establish corresponding boundedness of the multilinear commutator $L_{\vec{b}}^{-\alpha / 2} f$ on the weighted Morrey space, where $\vec{b}=\left(b_{1}, \ldots, b_{m}\right), b_{j} \in \mathrm{BMO}(w)$ ?

The question is not motivated only by a mere quest to extend the multilinear commutator $L_{\vec{b}}^{-\alpha / 2} f$ from the classical commutator $\left[b, I_{\alpha}\right]$ but rather by their natural appearance in analysis (see [3]).

To state the main results, we now give some definitions and notations.
A weight is a locally integrable function on $\mathbb{R}^{n}$ which takes values in $(0, \infty)$ almost everywhere. For a weight $w$ and a measurable set $E$, we define $w(E)=\int_{E} w(x) d x$, the Lebesgue measure of $E$, by $|E|$ and the characteristic function of $E$ by $\chi_{E}$. For a real number $p, 1<p<\infty$; $p^{\prime}$ is the conjugate of $p$; that is, $1 / p+1 / p^{\prime}=1$. The letter $C$ denotes a positive constant that may vary at each occurrence but is independent of the essential variable.

Definition 3 (see [7]). Let $1 \leq p<\infty, 0<\kappa<1$; let $w$ be a weight; then weighted Morrey space is defined by

$$
L^{p, \kappa}(w):=\left\{f \in L_{\mathrm{loc}}^{p}(w):\|f\|_{L^{p, \kappa}(w)}<\infty\right\},
$$

where

$$
\|f\|_{L^{p, \kappa}(w)}=\sup _{B}\left(\frac{1}{w(B)^{\kappa}} \int_{B}|f(x)|^{p} w(x) d x\right)^{1 / p},
$$

and the supremum is taken over all balls $B$ in $\mathbb{R}^{n}$.

Definition 4 (see [7]). Let $1 \leq p<\infty, 0<\kappa<1$; let $u, v$ be weight; then two weights weighted Morrey space are defined by

$$
L^{p, \kappa}(u, v):=\left\{f:\|f\|_{L^{p, \kappa}(u, v)}<\infty\right\}
$$

where

$$
\|f\|_{L^{p, \kappa}(u, v)}=\sup _{B}\left(\frac{1}{v(B)^{\kappa}} \int_{B}|f(x)|^{p} u(x) d x\right)^{1 / p},
$$

and the supremum is taken over all balls $B$ in $\mathbb{R}^{n}$. If $u=v$, then we denote $L^{p, \kappa}(u)$ for short.

Remark 5. (1) If $w=1, \kappa=\lambda / n$, and $0<\lambda<n$, then $L^{p, \kappa}(w)=L^{p, \lambda}\left(\mathbb{R}^{n}\right)$, the classical Morrey space.

(2) If $\kappa=0$, then $L^{p, 0}(w)=L^{p}(w)$, the weighted Lebesgue space; if $w=1, \kappa=0$, then $L^{p, \kappa}(w)=L^{p}\left(\mathbb{R}^{n}\right)$, the Lebesgue space.

Definition 6 (see [8]). A weight function $w$ is in the Muckenhoupt class $A_{p}$ with $1<p<\infty$ if for every ball $B$ in $\mathbb{R}^{n}$, there exists a positive constant $C$ which is independent of $B$ such that

$$
\left(\frac{1}{|B|} \int_{B} w(x) d x\right)\left(\frac{1}{|B|} \int_{B} w(x)^{-1 /(p-1)} d x\right)^{p-1} \leq C .
$$

When $p=1, w \in A_{1}$, if

$$
\frac{1}{|B|} \int_{B} w(x) d x \leq C \operatorname{ess} \inf _{x \in B} w(x) .
$$

When $p=\infty, w \in A_{\infty}$, if there exist positive constants $\delta$ and $C$ such that given a ball $B$ and $E$ is a measurable subset of $B$, then

$$
\frac{w(E)}{w(B)} \leq C\left(\frac{|E|}{|B|}\right)^{\delta}
$$


Definition 7 (see [9]). A weight function $w$ belongs to the reverse Hölder class $\mathrm{RH}_{r}$, if there exist two constants $r>1$ and $C>0$ such that the following reverse Hölder inequality

$$
\left(\frac{1}{|B|} \int_{B} w(x)^{r} d x\right)^{1 / r} \leq C \frac{1}{|B|} \int_{B} w(x) d x,
$$

holds for every ball $B$ in $\mathbb{R}^{n}$.

It is well known that if $w \in A_{p}$ with $1 \leq p<\infty$, then there exists $r>1$ such that $w \in \mathrm{RH}_{r}$. It follows from Hölder's inequality that $w \in \mathrm{RH}_{r}$ implies $w \in \mathrm{RH}_{s}$ for all $1<s<r$. Moreover, if $w \in \mathrm{RH}_{r}, r>1$, then we have $w \in \mathrm{RH}_{r+\varepsilon}$ for some $\varepsilon>0$. We thus write $r_{w} \equiv \sup \{r>1: w \epsilon$ $\left.\mathrm{RH}_{r}\right\}$ to denote the critical index of $w$ for the reverse Hölder condition.

Definition 8. The Hardy-Littlewood maximal operator $M$ is defined by

$$
M f(x)=\sup _{x \in B} \frac{1}{|B|} \int_{B}|f(y)| d y .
$$

Let $w$ be a weight. The weighted maximal operator $M_{w}$ is defined by

$$
M_{w} f(x)=\sup _{x \in B} \frac{1}{w(B)} \int_{B}|f(y)| w(y) d y .
$$

For $0<\alpha<n, r \geq 1$, the fractional maximal operator $M_{\alpha, r}$ is defined by

$$
M_{\alpha, r} f(x)=\sup _{x \in B}\left(\frac{1}{|B|^{1-\alpha r / n}} \int_{B}|f(y)|^{r} d y\right)^{1 / r} .
$$

And the fractional weighted maximal operator $M_{\alpha, r, w}$ is defined by

$$
M_{\alpha, r, w} f(x)=\sup _{x \in B}\left(\frac{1}{w(B)^{1-\alpha r / n}} \int_{B}|f(y)|^{r} w(y) d y\right)^{1 / r} .
$$

If $\alpha=0$, we denote $M_{r, w}$ for short.

Definition 9. A family of operators $\left\{A_{t}: t>0\right\}$ is said to be an "approximation to identity" if, for every $t>0, A_{t}$ is represented by the kernel $a_{t}(x, y)$, which is a measurable function defined on $\mathbb{R}^{n} \times \mathbb{R}^{n}$, in the following sense: for every $f \in L^{p}\left(\mathbb{R}^{n}\right), p \geq 1$,

$$
\begin{gathered}
A_{t} f(x)=\int_{\mathbb{R}^{n}} a_{t}(x, y) f(y) d y, \\
\left|a_{t}(x, y)\right| \leq h_{t}(x, y)=t^{-n / 2} g\left(\frac{|x-y|^{2}}{t}\right),
\end{gathered}
$$

for $(x, y) \in \mathbb{R}^{n} \times \mathbb{R}^{n}, t>0$. Here, $g$ is a positive, bounded, decreasing function satisfying

$$
\lim _{r \rightarrow \infty} r^{n+\varepsilon} g\left(r^{2}\right)=0
$$

for some $\varepsilon>0$.
Associated with an "approximation to identity" $\left\{A_{t}: t>\right.$ $0\}$, Martell [10] introduced the sharp maximal function as follows:

$$
M_{A}^{\sharp} f(x)=\sup _{x \in B} \frac{1}{|B|} \int_{B}\left|f(y)-A_{t_{B}} f(y)\right| d y,
$$

where $t_{B}=r_{B}^{2}, r_{B}$ is the radius of the ball $B$, and $f \in L^{p}\left(\mathbb{R}^{n}\right)$ for some $p \geq 1$.

Notice that our analytic semigroup $\left\{e^{-t L}: t>0\right\}$ is an “approximation to identity." In particular, denote

$$
M_{L}^{\sharp} f(x)=\sup _{x \in B} \frac{1}{|B|} \int_{B}\left|f(y)-e^{-t_{B} L} f(y)\right| d y .
$$

Next, we make some conventions on notation. Given any positive integer $m$, for all $0 \leq j \leq m$, we denote by $C_{j}^{m}$ the family of all finite subsets $\sigma=\left\{\sigma_{1}, \sigma_{2}, \ldots, \sigma_{j}\right\}$ of $\{1,2, \ldots, m\}$ of different elements, and, for any $\sigma \in C_{j}^{m}$, let $\sigma^{\prime}=\{1,2, \ldots, m\} \backslash \sigma$. Let $\vec{b}=\left(b_{1}, b_{2}, \ldots, b_{m}\right)$; then, for any $\sigma=\left\{\sigma_{1}, \sigma_{2}, \ldots, \sigma_{j}\right\} \in C_{j}^{m}$, we denote $\vec{b}_{\sigma}=\left(b_{\sigma_{1}}, b_{\sigma_{2}}, \ldots, b_{\sigma_{j}}\right)$, $b_{\sigma}(x)=\prod_{\sigma_{j} \in \sigma} b_{\sigma_{j}}(x)$ and $\left\|\vec{b}_{\sigma}\right\|_{\mathrm{BMO}(w)}=\prod_{\sigma_{j} \in \sigma}\left\|b_{\sigma_{j}}\right\|_{\mathrm{BMO}(w)}$, and $\|\vec{b}\|_{\mathrm{BMO}(w)}=\prod_{\sigma_{j} \in\{1,2, \ldots, m\}}\left\|b_{\sigma_{j}}\right\|_{\mathrm{BMO}(w)}=\prod_{j=1}^{m}\left\|b_{j}\right\|_{\mathrm{BMO}(w)}$.

In this paper, our main results are stated as follows.

Theorem 10. Assume the condition (1) holds. Let $0<\alpha<n$, $1<p<n / \alpha, 1 / q=1 / p-\alpha / n, 0 \leq \kappa<p / q, w^{q / p} \in A_{1}$, and $r_{w}>(1-\kappa) /(p / q-\kappa)$, where $r_{w}$ denotes the critical index of $w$ for the reverse Hölder condition. If $b_{j} \in B M O(w), j=$ $1,2, \ldots, m$, then

$$
\left\|L_{\vec{b}}^{-\alpha / 2} f\right\|_{L^{q, \kappa q / p}\left(w^{q / p}, w\right)} \leq C\|\vec{b}\|_{B M O(w)}\|f\|_{L^{p, \kappa}(w)} .
$$

Theorem 11. Let $0<\alpha<n, 1<p<n / \alpha, 1 / q=1 / p-\alpha / n$, $w^{q / p} \in A_{1}$, and $r_{w}>q / p$, where $r_{w}$ denotes the critical index of $w$ for the reverse Hölder condition. If $b_{j} \in B M O(w), j=$ $1,2, \ldots, m$, then

$$
\left\|L_{\vec{b}}^{-\alpha / 2} f\right\|_{L^{q}\left(w^{q / p}\right)} \leq C\|\vec{b}\|_{B M O(w)}\|f\|_{L^{p}(w)} .
$$

Moreover, if $L=-\Delta$ is the Laplacian, then

$$
\left\|I_{\alpha}^{\vec{b}} f\right\|_{L^{q}\left(w^{q / p}\right)} \leq C\|\vec{b}\|_{B M O(w)}\|f\|_{L^{p}(w)} .
$$

Remark 12. We note that our results extend some results in $[1,3]$. To be specific, if we take $m=1, w=1$, and $\kappa=0$ in Theorem 10, it is easy to see that our conclusion is the main result of Duong and Yan [1]. If we only take $w=1$ and $\kappa=0$ in Theorem 10, our result contains the corresponding conclusion of [3].

The remaining part of this paper will be organized as follows. In Section 2, we will give some known results and prove some requisite lemmas. Section 3 is devoted to proving the theorems of this paper. 


\section{Requisite Lemmas}

In this section, we will prove some lemmas and state some known results about weights and weighted Morrey space.

Lemma 13 (see [11]). Let $s>1,1 \leq p<\infty$, and $A_{p}^{s}=\{w$ : $\left.w^{s} \in A_{p}\right\}$. Then

$$
A_{p}^{s}=A_{1+(p-1) / s} \cap R H_{s} .
$$

In particular, $A_{1}^{s}=A_{1} \cap R H_{s}$.

Lemma 14 (see [10]). Assume that the semigroup $e^{-t L}$ has a kernel $p_{t}(x, y)$ which satisfies the upper bound (1). Take $\lambda>0$, $f \in L_{0}^{1}\left(\mathbb{R}^{n}\right)$ (the set of functions in $L^{1}\left(\mathbb{R}^{n}\right)$ with bounded support) and a ball $B_{0}$ such that there exists $x_{0} \in B_{0}$ with $M f\left(x_{0}\right) \leq \lambda$. Then, for every $w \in A_{\infty}, 0<\eta<1$, we can find $\gamma>0$ (independent of $\lambda, B_{0}, f, x_{0}$ ) and constants $C, r>0$ (which only depend on $w$ ) such that

$$
w\left(\left\{x \in B_{0}: M f(x)>A \lambda, M_{L}^{\sharp} f(x) \leq \gamma \lambda\right\}\right) \leq C \eta^{r} w\left(B_{0}\right),
$$

where $A>1$ is a fixed constant which depends only on $n$.

As a result, using the above good- $\lambda$ inequality together with the standard arguments, we have the following estimates:

For every $f \in L^{p, \kappa}(u, v), 1<p<\infty, 0 \leq \kappa<1$; if $u, v \in A_{\infty}$, then

$$
\|f\|_{L^{p, \kappa}(u, v)} \leq\|M f\|_{L^{p, \kappa}(u, v)} \leq\left\|M_{L}^{\sharp} f\right\|_{L^{p, \kappa}(u, v)} .
$$

In particular, when $u=v=w, w \in A_{\infty}$, we have

$$
\|f\|_{L^{p, \kappa}(w)} \leq\|M f\|_{L^{p, \kappa}(w)} \leq\left\|M_{L}^{\sharp} f\right\|_{L^{p, \kappa}(w)} .
$$

Lemma 15 (Kolmogorov's inequality; see [9, page 455]). Let $0<r<l<\infty$, for $f \geq 0$; define $\|f\|_{L^{l, \infty}}=\sup _{t>0} t \mid\{x \in$ $\left.\mathbb{R}^{n}:|f(x)|>t\right\}\left.\right|^{1 / l}, N_{l, r}(f)=\sup _{E}\left(\left\|f \chi_{E}\right\|_{r} /\left\|\chi_{E}\right\|_{h}\right)$, and $1 / h=$ $1 / r-1 / l ;$ then

$$
\|f\|_{L^{l, \infty}} \leq N_{l, r}(f) \leq\left(\frac{l}{l-r}\right)^{1 / r}\|f\|_{L^{l, \infty}} .
$$

Lemma 16 (see [4]). Let $w \in A_{\infty}$. Then the norm of $B M O(w)$ : $\|\cdot\|_{*, w}$, is equivalent to the norm of $B M O\left(\mathbb{R}^{n}\right):\|\cdot\|_{*}$; that is, if $b$ is a locally integrable function, then

$$
\|b\|_{*, w}=\sup _{\mathrm{Q}} \frac{1}{w(Q)} \int_{\mathrm{Q}}\left|b(x)-b_{\mathrm{Q}, w}\right| w(x) d x \leq C
$$

is equivalent to

$$
\|b\|_{*}=\sup _{\mathrm{Q}} \frac{1}{|Q|} \int_{\mathrm{Q}}\left|b(x)-b_{\mathrm{Q}}\right| d x \leq C .
$$

Lemma 17 (see [5]). Let $0<\alpha<n, 1<p<n / \alpha, 1 / q=1 / p-$ $\alpha / n$, and $w^{q / p} \in A_{1}$; if $0<\kappa<p / q, r_{w}>(1-\kappa) /(p / q-\kappa)$, then

$$
\left\|M_{\alpha, 1} f\right\|_{L^{q, \kappa q / p}\left(w^{q / p}, w\right)} \leq C\|f\|_{L^{p, \kappa}(w)} .
$$

It also holds for $I_{\alpha}$.
Lemma 18 (see [5]). Let $0<\alpha<n, 1<p<n / \alpha, 1 / q=$ $1 / p-\alpha / n$, and $w^{q / p} \in A_{1}$; if $0<\kappa<p / q, 1<r<p$, $r_{w}>(1-\kappa) /(p / q-\kappa)$, then

$$
\left\|M_{r, w} f\right\|_{L^{q, \kappa q / p}\left(w^{q / p}, w\right)} \leq C\|f\|_{L^{q, \kappa q / p}\left(w^{q / p}, w\right)} .
$$

Lemma 19 (see [5]). Let $0<\alpha<n, 1<p<n / \alpha, 1 / q=$ $1 / p-\alpha / n, 0<\kappa<p / q$, and $w \in A_{\infty}$. Then, for $1<r<p$,

$$
\left\|M_{\alpha, r, w} f\right\|_{L^{q, k q / p}(w)} \leq C\|f\|_{L^{p, \kappa}(w)} .
$$

Remark 20. It is easy to see that Lemmas 17, 18, and 19 still hold for $\kappa=0$.

Lemma 21. Let $0<\alpha<n, 1<p<n / \alpha, 1 / q=1 / p-\alpha / n$, and $w^{q / p} \in A_{1}$; if $0 \leq \kappa<p / q, r_{w}>(1-\kappa) /(p / q-\kappa)$, then

$$
\left\|L^{-\alpha / 2} f\right\|_{L^{q, \kappa q / p}\left(w^{q / p}, w\right)} \leq C\|f\|_{L^{p, \kappa}(w)} .
$$

Proof. Since semigroup $e^{-t L}$ has a kernel $p_{t}(x, y)$ that satisfies the upper bound (1), it is easy to see that, for $x \in \mathbb{R}^{n}$, $L^{-\alpha / 2} f(x) \leq I_{\alpha}(|f|)(x)$. From the boundedness of $I_{\alpha}$ on weighted Morrey space (see Lemma 17), we get

$$
\left\|L^{-\alpha / 2} f\right\|_{L^{q, \kappa q / p}\left(w^{q / p}, w\right)} \leq\left\|I_{\alpha} f\right\|_{L^{q, \kappa q / p}\left(w^{q / p}, w\right)} \leq C\|f\|_{L^{p, \kappa}(w)} .
$$

Remark 22. Since $I_{\alpha}$ is of weak $(1, n /(n-\alpha))$ type, from the above proof, we can obtain that $L^{-\alpha / 2}$ is of weak $(1, n /(n-\alpha))$ type.

Lemma 23 (see [1]). Assume the semigroup $e^{-t L}$ has a kernel $p_{t}(x, y)$ which satisfies the upper bound (1). Then, for $0<\alpha<$ $n$, the differential operator $L^{-\alpha / 2}-e^{-t L} L^{-\alpha / 2}$ has an associated kernel $\widetilde{K}_{\alpha, t}(x, y)$ which satisfies

$$
\widetilde{K}_{\alpha, t}(x, y) \leq \frac{C}{|x-y|^{n-\alpha}} \frac{t}{|x-y|^{2}} .
$$

Lemma 24. Assume the semigroup $e^{-t L}$ has a kernel $p_{t}(x, y)$ which satisfies the upper bound (1), $b \in B M O(w)$, and $w \in A_{1}$. Then, for $f \in L^{p}\left(\mathbb{R}^{n}\right), p>1, \sigma \in C_{j}^{m}(j=1,2, \ldots, m), 1<$ $\tau<\infty$, and

$$
\begin{gathered}
\sup _{x \in B} \frac{1}{|B|} \int_{B}\left|e^{-t_{B} L}\left(\left(b-b_{B}\right)_{\sigma} f\right)(y)\right| d y \\
\leq C\left\|\vec{b}_{\sigma}\right\|_{B M O(w)} M_{\tau, w} f(x),
\end{gathered}
$$

where $t_{B}=r_{B}^{2}$ and $r_{B}$ is the radius of $B$. 
Proof. For any $f \in L^{p}\left(\mathbb{R}^{n}\right), x \in B$, we have

$$
\begin{aligned}
& \frac{1}{|B|} \int_{B}\left|e^{-t_{B} L}\left(\left(b-b_{B}\right)_{\sigma} f\right)(y)\right| d y \\
& \leq \frac{1}{|B|} \int_{B} \int_{\mathbb{R}^{n}}\left|p_{t_{B}}(y, z)\right|\left|\left(b(z)-b_{B}\right)_{\sigma} f(z)\right| d z d y \\
& \leq \frac{1}{|B|} \int_{B} \int_{2 B}\left|p_{t_{B}}(y, z)\right|\left|\left(b(z)-b_{B}\right)_{\sigma} f(z)\right| d z d y \\
& \quad+\frac{1}{|B|} \int_{B} \sum_{k=1}^{\infty} \int_{2^{k+1} B \backslash 2^{k} B}\left|p_{t_{B}}(y, z)\right| \\
& \quad \times\left|\left(b(z)-b_{B}\right)_{\sigma} f(z)\right| d z d y \\
& :=I+I I . \quad
\end{aligned}
$$

Noticing that $y \in B, z \in 2 B$, from (1), we get

$$
\left|p_{t_{B}}(y, z)\right| \leq C t_{B}^{-n / 2} \leq C|2 B|^{-1} .
$$

Thus,

$$
\begin{aligned}
I & \leq \frac{C}{|2 B|} \int_{2 B}\left|\left(b(z)-b_{B}\right)_{\sigma}\right||f(z)| d z \\
& =\frac{C}{|2 B|} \int_{2 B} \prod_{\sigma_{j} \in \sigma}\left|b_{\sigma_{j}}(z)-\left(b_{\sigma_{j}}\right)_{B}\right||f(z)| d z .
\end{aligned}
$$

For simplicity, we only consider the case of $j=2$. We also want to point out that although we state our results on the case of $j=2$, all results are valid on the multilinear case $(j>2)$ without any essential difference and difficulty in the proof. So it follows that

$$
\begin{aligned}
I \leq & \frac{C}{|2 B|} \int_{2 B}\left(\left|b_{\sigma_{1}}(z)-\left(b_{\sigma_{1}}\right)_{2 B}\right|+\left|\left(b_{\sigma_{1}}\right)_{2 B}-\left(b_{\sigma_{1}}\right)_{B}\right|\right) \\
& \times\left(\left|b_{\sigma_{2}}(z)-\left(b_{\sigma_{2}}\right)_{2 B}\right|+\left|\left(b_{\sigma_{2}}\right)_{2 B}-\left(b_{\sigma_{2}}\right)_{B}\right|\right)|f(z)| d z \\
\leq & \frac{C}{|2 B|} \int_{2 B}\left|b_{\sigma_{1}}(z)-\left(b_{\sigma_{1}}\right)_{2 B}\right|\left|b_{\sigma_{2}}(z)-\left(b_{\sigma_{2}}\right)_{2 B}\right||f(z)| d z \\
& +\frac{C}{|2 B|} \int_{2 B}\left|b_{\sigma_{1}}(z)-\left(b_{\sigma_{1}}\right)_{2 B}\right|\left|\left(b_{\sigma_{2}}\right)_{2 B}-\left(b_{\sigma_{2}}\right)_{B}\right||f(z)| d z \\
& +\frac{C}{|2 B|} \int_{2 B}\left|\left(b_{\sigma_{1}}\right)_{2 B}-\left(b_{\sigma_{1}}\right)_{B}\right|\left|b_{\sigma_{2}}(z)-\left(b_{\sigma_{2}}\right)_{2 B}\right||f(z)| d z \\
& +\frac{C}{|2 B|} \int_{2 B}\left|\left(b_{\sigma_{1}}\right)_{2 B}-\left(b_{\sigma_{1}}\right)_{B}\right|\left|\left(b_{\sigma_{2}}\right)_{2 B}-\left(b_{\sigma_{2}}\right)_{B}\right||f(z)| d z \\
:= & I_{1}+I_{2}+I_{3}+I_{4} .
\end{aligned}
$$

We split $I_{1}$ as follows:

$$
\begin{aligned}
I_{1} \leq \frac{C}{|2 B|} \int_{2 B} & \left\{\left|b_{\sigma_{1}}(z)-\left(b_{\sigma_{1}}\right)_{2 B, w}\right|+\left|\left(b_{\sigma_{1}}\right)_{2 B, w}-\left(b_{\sigma_{1}}\right)_{2 B}\right|\right\} \\
\times & \left\{\left|b_{\sigma_{2}}(z)-\left(b_{\sigma_{2}}\right)_{2 B, w}\right|\right. \\
+ & \left.\left|\left(b_{\sigma_{2}}\right)_{2 B, w}-\left(b_{\sigma_{2}}\right)_{2 B}\right|\right\}|f(z)| d z
\end{aligned}
$$

$$
\begin{aligned}
& =\frac{C}{|2 B|} \int_{2 B}\left|b_{\sigma_{1}}(z)-\left(b_{\sigma_{1}}\right)_{2 B, w}\right| \\
& \times\left|b_{\sigma_{2}}(z)-\left(b_{\sigma_{2}}\right)_{2 B, w}\right||f(z)| d z \\
& +\frac{C}{|2 B|} \int_{2 B}\left|b_{\sigma_{1}}(z)-\left(b_{\sigma_{1}}\right)_{2 B, w}\right| \\
& \times\left|\left(b_{\sigma_{2}}\right)_{2 B, w}-\left(b_{\sigma_{2}}\right)_{2 B}\right||f(z)| d z \\
& +\frac{C}{|2 B|} \int_{2 B}\left|\left(b_{\sigma_{1}}\right)_{2 B, w}-\left(b_{\sigma_{1}}\right)_{2 B}\right| \\
& \times\left|b_{\sigma_{2}}(z)-\left(b_{\sigma_{2}}\right)_{2 B, w}\right||f(z)| d z \\
& +\frac{C}{|2 B|} \int_{2 B}\left|\left(b_{\sigma_{1}}\right)_{2 B, w}-\left(b_{\sigma_{1}}\right)_{2 B}\right| \\
& \times\left|\left(b_{\sigma_{2}}\right)_{2 B, w}-\left(b_{\sigma_{2}}\right)_{2 B}\right||f(z)| d z \\
& :=I_{11}+I_{12}+I_{13}+I_{14} \text {. }
\end{aligned}
$$

We now consider the four terms, respectively. Choose $\tau_{1}, \tau_{2}, \tau, s>1$ satisfing $1 / \tau_{1}+1 / \tau_{2}+1 / \tau+1 / s=1$. According to Hölder's inequality and $w \in A_{1}$, we have

$$
\begin{aligned}
& I_{11}=\frac{C}{|2 B|} \int_{2 B}\left|b_{\sigma_{1}}(z)-\left(b_{\sigma_{1}}\right)_{2 B, w}\right| w(z)^{1 / \tau_{1}} \\
& \quad \times\left|b_{\sigma_{2}}(z)-\left(b_{\sigma_{2}}\right)_{2 B, w}\right| w(z)^{1 / \tau_{2}}|f(z)| \\
& \quad \times w(z)^{1 / \tau} w(z)^{-1+1 / s} d z \\
& \leq \frac{C}{|2 B|}\left(\int_{2 B}\left|b_{\sigma_{1}}(z)-\left(b_{\sigma_{1}}\right)_{2 B, w}\right|^{\tau_{1}} w(z) d z\right)^{1 / \tau_{1}} \\
& \quad \times\left(\int_{2 B}\left|b_{\sigma_{2}}(z)-\left(b_{\sigma_{2}}\right)_{2 B, w}\right|^{\tau_{2}} w(z) d z\right)^{1 / \tau_{2}} \\
& \quad \times\left(\int_{2 B}|f(z)|^{\tau} w(z) d z\right)^{1 / \tau}\left(\int_{2 B} w(z)^{-s+1} d z\right)^{1 / s} \\
& \leq \frac{C}{|2 B|}\left\|\vec{b}_{\sigma}\right\|_{*, w} M_{\tau, w} f(x) w(2 B)^{1-1 / s} w(x)^{-1+1 / s}|2 B|^{1 / s} \\
&= C\left\|\vec{b}_{\sigma}\right\|_{*} M_{\tau, w} f(x)\left(\frac{w(2 B)}{|2 B|}\right)^{1-1 / s} w(x)^{-1+1 / s} \\
& \leq C\left\|\vec{b}_{\sigma}\right\|_{*} M_{\tau, w} f(x) .
\end{aligned}
$$

In the above inequalities, we use the fact that if $w \in A_{1}$, then $w \in A_{\infty}$. Thus, the norm of $\operatorname{BMO}(w)$ is equivalent to the norm of $\operatorname{BMO}\left(\mathbb{R}^{n}\right)$ (see Lemma 16 ).

For $I_{12}$, we first estimate the term that contains $\left(b_{\sigma_{2}}\right)_{2 B}$. In fact, it follows from the John-Nirenberg lemma that there exist $C_{1}>0$ and $C_{2}>0$ such that, for any ball $B$ and $\alpha>0$,

$$
\left|\left\{z \in 2 B:\left|b_{\sigma_{2}}(z)-\left(b_{\sigma_{2}}\right)_{2 B}\right|>\alpha\right\}\right| \leq C_{1}|2 B| e^{-C_{2} \alpha /\left\|b_{\sigma_{2}}\right\|_{*}},
$$


since $b \in \mathrm{BMO}\left(\mathbb{R}^{n}\right)$. Using the definition of $A_{\infty}$, we get

$$
\begin{aligned}
& w\left(\left\{z \in 2 B:\left|b_{\sigma_{2}}(z)-\left(b_{\sigma_{2}}\right)_{2 B}\right|>\alpha\right\}\right) \\
& \quad \leq C w(2 B) e^{-C_{2} \alpha \delta /\left\|b_{\sigma_{2}}\right\|_{*}},
\end{aligned}
$$

for some $\delta>0$. We can see that (48) yields

$$
\begin{aligned}
& \int_{2 B}\left|b_{\sigma_{2}}(z)-\left(b_{\sigma_{2}}\right)_{2 B}\right| w(z) d z \\
& =\int_{0}^{\infty} w\left(\left\{z \in 2 B:\left|b_{\sigma_{2}}(z)-\left(b_{\sigma_{2}}\right)_{2 B}\right|>\alpha\right\}\right) d \alpha \\
& \leq C w(2 B) \int_{0}^{\infty} e^{-C_{2} \alpha \delta /\left\|b_{\sigma_{2}}\right\|_{*}} d \alpha \\
& \quad=C w(2 B)\left\|b_{\sigma_{2}}\right\|_{*} .
\end{aligned}
$$

Thus,

$$
\begin{aligned}
& \left|\left(b_{\sigma_{2}}\right)_{2 B, w}-\left(b_{\sigma_{2}}\right)_{2 B}\right| \\
& \quad \leq \frac{1}{w(2 B)} \int_{2 B}\left|b_{\sigma_{2}}(z)-\left(b_{\sigma_{2}}\right)_{2 B}\right| w(z) d z \\
& \quad \leq \frac{C}{w(2 B)} w(2 B)\left\|b_{\sigma_{2}}\right\|_{*}=C\left\|b_{\sigma_{2}}\right\|_{*} .
\end{aligned}
$$

On the basis of (50), we now estimate $I_{12}$. For the above $\tau$, select $u, v$ such that $1 / u+1 / v+1 / \tau=1$; by virtue of Hölder's inequality and $w \in A_{1}$, we have

$$
\begin{aligned}
I_{12} \leq & C\left\|b_{\sigma_{2}}\right\|_{*} \frac{1}{|2 B|} \int_{2 B}\left|b_{\sigma_{1}}(z)-\left(b_{\sigma_{1}}\right)_{2 B, w}\right||f(z)| d z \\
= & C\left\|b_{\sigma_{2}}\right\|_{*} \frac{1}{|2 B|} \int_{2 B}\left|b_{\sigma_{1}}(z)-\left(b_{\sigma_{1}}\right)_{2 B, w}\right| \\
& \times w(z)^{1 / u}|f(z)| w(z)^{1 / \tau} w(z)^{-1+1 / v} d z \\
\leq & C\left\|b_{\sigma_{2}}\right\|_{*} \frac{1}{|2 B|}\left(\int_{2 B}\left|b_{\sigma_{1}}(z)-\left(b_{\sigma_{1}}\right)_{2 B, w}\right|^{u} w(z) d z\right)^{1 / u} \\
& \times\left(\int_{2 B}|f(z)|^{\tau} w(z) d z\right)^{1 / \tau}\left(\int_{2 B} w(z)^{-v+1} d z\right)^{1 / v} \\
\leq & C\left\|\vec{b}_{\sigma}\right\|_{*} M_{\tau, w} f(x) \frac{1}{|2 B|} w(2 B)^{1 / u+1 / \tau} w(x)^{-1+1 / v}|2 B|^{1 / v} \\
= & C\left\|\vec{b}_{\sigma}\right\|_{*} M_{\tau, w} f(x)\left(\frac{w(2 B)}{|2 B|}\right)^{1-1 / v} w(x)^{-1+1 / v} \\
= & C\left\|\vec{b}_{\sigma}\right\|_{*} M_{\tau, w} f(x) .
\end{aligned}
$$

Analogous to the estimate of $I_{12}$, we also have $I_{13} \leq$ $C\left\|\vec{b}_{\sigma}\right\|_{*} M_{\tau, w} f(x)$.
As for $I_{14}$, taking advantage of Hölder's inequality and (50), we get

$$
\begin{aligned}
I_{14} \leq & C\left\|\vec{b}_{\sigma}\right\|_{*} \frac{1}{|2 B|} \int_{2 B}|f(z)| d z \\
= & C\left\|\vec{b}_{\sigma}\right\|_{*} \frac{1}{|2 B|} \int_{2 B}|f(z)| w(z)^{1 / \tau} w(z)^{1 / \tau^{\prime}-1} d z \\
\leq & C\left\|\vec{b}_{\sigma}\right\|_{*} \frac{1}{|2 B|}\left(\int_{2 B}|f(z)|^{\tau} w(z) d z\right)^{1 / \tau} \\
& \times\left(\int_{2 B} w(z)^{1-\tau^{\prime}} d z\right)^{1 / \tau^{\prime}} \\
= & C\left\|\vec{b}_{\sigma}\right\|_{*} \frac{1}{|2 B|} M_{\tau, w} f(x) w(2 B)^{1 / \tau} w(x)^{1 / \tau^{\prime}-1}|2 B|^{1 / \tau^{\prime}} \\
= & C\left\|\vec{b}_{\sigma}\right\|_{*} M_{\tau, w} f(x)\left(\frac{w(2 B)}{|2 B|}\right)^{1 / \tau} w(x)^{1 / \tau^{\prime}-1} \\
\leq & C\left\|\vec{b}_{\sigma}\right\|_{*} M_{\tau, w} f(x) .
\end{aligned}
$$

Collecting the estimates of $I_{11}, I_{12}, I_{13}$, and $I_{14}$, it is easy to see that

$$
I_{1} \leq C\left\|\vec{b}_{\sigma}\right\|_{*} M_{\tau, w} f(x) .
$$

For term $I_{2}$, using the fact that $\left|b_{2 B}-b_{B}\right| \leq C\|b\|_{*}$ (see [12]), we now get

$$
\begin{aligned}
I_{2} \leq & \left\|b_{\sigma_{2}}\right\|_{*} \frac{C}{|2 B|} \int_{2 B}\left(\left|b_{\sigma_{1}}(z)-\left(b_{\sigma_{1}}\right)_{2 B, w}\right|\right. \\
& \left.+\left|\left(b_{\sigma_{1}}\right)_{2 B, w}-\left(b_{\sigma_{1}}\right)_{2 B}\right|\right) \\
& \times|f(z)| d z \\
= & \frac{C\left\|b_{\sigma_{2}}\right\|_{*}}{|2 B|} \int_{2 B}\left|b_{\sigma_{1}}(z)-\left(b_{\sigma_{1}}\right)_{2 B, w}\right||f(z)| d z \\
& +\frac{C\left\|b_{\sigma_{2}}\right\|_{*}}{|2 B|} \int_{2 B}\left|\left(b_{\sigma_{1}}\right)_{2 B, w}-\left(b_{\sigma_{1}}\right)_{2 B}\right||f(z)| d z \\
:= & I_{21}+I_{22} .
\end{aligned}
$$

By some estimates similar to those used in the estimate for $I_{12}$, we conclude that

$$
I_{21} \leq C\left\|b_{\sigma}\right\|_{*} M_{\tau, w} f(x) .
$$

For $I_{22}$, using the same method as in dealing with $I_{14}$, we get

$$
I_{22} \leq C\left\|b_{\sigma}\right\|_{*} M_{\tau, w} f(x) \text {. }
$$

Therefore,

$$
I_{2} \leq C\left\|b_{\sigma}\right\|_{*} M_{\tau, w} f(x) .
$$

Analogously,

$$
I_{3} \leq C\left\|b_{\sigma}\right\|_{*} M_{\tau, w} f(x) .
$$


An argument similar to that used in the estimate for $I_{14}$ leads to

$$
I_{4} \leq C\left\|b_{\sigma}\right\|_{*} M_{\tau, w} f(x) .
$$

Hence,

$$
\begin{aligned}
I & \leq I_{1}+I_{2}+I_{3}+I_{4} \\
& \leq C\left\|b_{\sigma}\right\|_{*} M_{\tau, w} f(x) .
\end{aligned}
$$

Next, we will consider the second term $I I$ in (41). For any $y \in B, z \in 2^{k+1} B \backslash 2^{k} B$, it is easy to get that $|y-z| \geq 2^{k-1} r_{B}$ and

$$
\left|p_{t_{B}}(y, z)\right| \leq C \frac{e^{-C 2^{2(k-1)}} 2^{(k+1) n}}{\left|2^{k+1} B\right|}
$$

Thus,

$$
\begin{aligned}
I I \leq & C \sum_{k=1}^{\infty} \frac{e^{-C 2^{2(k-1)}} 2^{(k+1) n}}{\left|2^{k+1} B\right|} \int_{2^{k+1} B}\left|\left(b(z)-b_{B}\right)_{\sigma}\right||f(z)| d z \\
= & C \sum_{k=1}^{\infty} \frac{e^{-C 2^{2(k-1)}} 2^{(k+1) n}}{\left|2^{k+1} B\right|} \\
& \times \int_{2^{k+1} B}\left|b_{\sigma_{1}}(z)-\left(b_{\sigma_{1}}\right)_{B}\right| \cdots\left|b_{\sigma_{j}}(z)-\left(b_{\sigma_{j}}\right)_{B}\right||f(z)| d z .
\end{aligned}
$$

For simplicity, we also consider the case of $j=2$.

$$
\begin{aligned}
I I \leq C \sum_{k=1}^{\infty} \frac{e^{-C 2^{2(k-1)}} 2^{(k+1) n}}{\left|2^{k+1} B\right|} \\
\quad \times \int_{2^{k+1} B}\left(\left|b_{\sigma_{1}}(z)-\left(b_{\sigma_{1}}\right)_{2^{k+1} B}\right|\right. \\
\left.+\left|\left(b_{\sigma_{1}}\right)_{2^{k+1} B}-\left(b_{\sigma_{1}}\right)_{B}\right|\right) \\
\quad \times\left(\left|b_{\sigma_{2}}(z)-\left(b_{\sigma_{2}}\right)_{2^{k+1} B}\right|\right. \\
=C \sum_{k=1}^{\infty} \frac{e^{-C 2^{2(k-1)} 2^{(k+1) n}}}{\left|2^{k+1} B\right|} \\
\left.\quad \times \int_{2^{k+1} B}\left|b_{\sigma_{1}}(z)-\left(b_{\sigma_{2}}\right)_{2^{k+1} B}-\left(b_{\sigma_{2}}\right)_{B}\right|\right)|f(z)| d z \\
\quad \times\left|b_{\sigma_{2}}(z)-\left(b_{\sigma_{2}}\right)_{2^{k+1} B}\right||f(z)| d z \\
+C \sum_{k=1}^{\infty} \frac{e^{-C 2^{2(k-1)}} 2^{(k+1) n}}{\left|2^{k+1} B\right|} \\
\quad \times \int_{2^{k+1} B}\left|\left(b_{\sigma_{1}}\right)_{2^{k+1} B}-\left(b_{\sigma_{1}}\right)_{B}\right| \\
\quad \times\left|b_{\sigma_{2}}(z)-\left(b_{\sigma_{2}}\right)_{2^{k+1} B}\right||f(z)| d z
\end{aligned}
$$

$$
\begin{aligned}
&+C \sum_{k=1}^{\infty} \frac{e^{-C 2^{2(k-1)}} 2^{(k+1) n}}{\left|2^{k+1} B\right|} \\
& \times \int_{2^{k+1} B}\left|b_{\sigma_{1}}(z)-\left(b_{\sigma_{1}}\right)_{2^{k+1} B}\right| \\
& \times\left|\left(b_{\sigma_{2}}\right)_{2^{k+1} B}-\left(b_{\sigma_{2}}\right)_{B}\right||f(z)| d z \\
&+C \sum_{k=1}^{\infty} \frac{e^{-C 2^{2(k-1)}} 2^{(k+1) n}}{\left|2^{k+1} B\right|} \\
& \quad \times \int_{2^{k+1} B}\left|\left(b_{\sigma_{1}}\right)_{2^{k+1} B}-\left(b_{\sigma_{1}}\right)_{B}\right| \\
& \quad \times\left|\left(b_{\sigma_{2}}\right)_{2^{k+1} B}-\left(b_{\sigma_{2}}\right)_{B}\right||f(z)| d z \\
&:=I I_{1}+I I_{2}+I I_{3}+I I_{4} .
\end{aligned}
$$

For $I I_{1}$, similar to the estimate of $I_{1}$, we have

$$
\begin{aligned}
I I_{1} & \leq C\left\|\vec{b}_{\sigma}\right\|_{*} M_{\tau, w} f(x) \sum_{k=1}^{\infty} e^{-C 2^{2(k-1)}} 2^{(k+1) n} \\
& \leq C\left\|\vec{b}_{\sigma}\right\|_{*} M_{\tau, w} f(x) .
\end{aligned}
$$

Noticing that we could use similar methods of the estimates of $I_{2}, I_{3}$, and $I_{4}$ in the estimates of $I I_{2}, I I_{3}$, and $I I_{4}$, respectively. From this together with the fact that $\left|b_{2^{j+1} B}-b_{B}\right| \leq 2^{n}(j+$ $1)\|b\|_{*}$ (see [12]), it is easy to get

$$
I I_{2}+I I_{3}+I I_{4} \leq C\left\|\vec{b}_{\sigma}\right\|_{*} M_{\tau, w} f(x) .
$$

Therefore,

$$
I I \leq C\left\|\vec{b}_{\sigma}\right\|_{*} M_{\tau, w} f(x) .
$$

According to the estimates of $I$ and $I I$, the lemma has been proved.

Now, we will establish a lemma which plays an important role in the proof of Theorem 10.

Lemma 25. Let $0<\alpha<n, w \in A_{1}$, and $b \in B M O(w)$; then, for all $r>1, \tau>1$, and $x \in \mathbb{R}^{n}$, we have

$$
\begin{aligned}
& M_{L}^{\sharp}\left(L_{\vec{b}}^{-\alpha / 2} f\right)(x) \\
& \leq C\left\{\|\vec{b}\|_{*} M_{r, w}\left(L^{-\alpha / 2} f\right)(x)\right. \\
& \quad+\sum_{j=1}^{m-1} \sum_{\sigma \in C_{j}^{m}} C_{j, m}\left\|\vec{b}_{\sigma}\right\|_{*} M_{\tau, w}\left(L_{\vec{b}_{\sigma^{\prime}}}^{-\alpha / 2} f\right)(x) \\
& \quad+\|\vec{b}\|_{*} w(x)^{-\alpha / n} M_{\alpha, r, w} f(x) \\
& \left.\quad+\|\vec{b}\|_{*} M_{\alpha, 1} f(x)\right\} .
\end{aligned}
$$

Proof. For any given $x \in \mathbb{R}^{n}$, take a ball $B=B\left(x_{0}, r_{B}\right)$ which contains $x$. For $f \in L^{p}\left(\mathbb{R}^{n}\right)$, let $f_{1}=f \chi_{2 B}, f_{2}=f-f_{1}$. 
Denote the kernel of $L^{-\alpha / 2}$ by $K_{\alpha}(x, y), \vec{\lambda}=\left(\lambda_{1}, \lambda_{2}, \ldots, \lambda_{m}\right)$, where $\lambda_{j} \in \mathbb{R}^{n}, j=1,2, \ldots, m$. Then $L_{\vec{b}}^{-\alpha / 2} f$ can be written in the following form:

$$
\begin{aligned}
& L_{\vec{b}}^{-\alpha / 2} f(y) \\
& =\int_{\mathbb{R}^{n}} \prod_{j=1}^{m}\left(b_{j}(y)-b_{j}(z)\right) K_{\alpha}(y, z) f(z) d z \\
& =\int_{\mathbb{R}^{n}} \prod_{j=1}^{m}\left(\left(b_{j}(y)-\lambda_{j}\right)-\left(b_{j}(z)-\lambda_{j}\right)\right) K_{\alpha}(y, z) f(z) d z \\
& =\sum_{i=0}^{m} \sum_{\sigma \in C_{i}^{m}}(-1)^{m-i}(b(y)-\lambda)_{\sigma} \\
& \quad \times \int_{\mathbb{R}^{n}}(b(z)-\lambda)_{\sigma^{\prime}} K_{\alpha}(y, z) f(z) d z .
\end{aligned}
$$

Now expanding $(b(z)-\lambda)_{\sigma^{\prime}}$ as

$$
(b(z)-\lambda)_{\sigma^{\prime}}=((b(z)-b(y))+(b(y)-\lambda))_{\sigma^{\prime}},
$$

and combining (68) with (69), it is easy to see that

$$
\begin{aligned}
& e^{-t_{B} L}\left(L_{\vec{b}}^{-\alpha / 2} f\right)(y) \\
& =e^{-t_{B} L}\left(\prod_{j=1}^{m}\left(b_{j}-\lambda_{j}\right) L^{-\alpha / 2} f\right)(y) \\
& \quad+\sum_{j=1}^{m-1} \sum_{\sigma \in C_{j}^{m}} C_{j, m} e^{-t_{B} L}\left((b-\lambda)_{\sigma} L_{\vec{b}_{\sigma^{\prime}}}^{-\alpha / 2} f\right)(y) \\
& \quad+(-1)^{m} e^{-t_{B} L}\left(L^{-\alpha / 2}\left(\prod_{j=1}^{m}\left(b_{j}-\lambda_{j}\right) f_{1}\right)\right)(y) \\
& \quad+(-1)^{m} e^{-t_{B} L}\left(L^{-\alpha / 2}\left(\prod_{j=1}^{m}\left(b_{j}-\lambda_{j}\right) f_{2}\right)\right)(y),
\end{aligned}
$$

where $t_{B}=r_{B}^{2}$ and $r_{B}$ is the radius of ball $B$.

Take $\lambda_{j}=\left(b_{j}\right)_{B}, j=1,2, \ldots, m$, and denote $\vec{b}_{B}=$ $\left(\left(b_{1}\right)_{B},\left(b_{2}\right)_{B}, \ldots,\left(b_{m}\right)_{B}\right)$; then

$$
\begin{aligned}
& \frac{1}{|B|} \int_{B}\left|L_{\vec{b}}^{-\alpha / 2} f(y)-e^{-t_{B} L}\left(L_{\vec{b}}^{-\alpha / 2} f\right)(y)\right| d y \\
& \leq \frac{1}{|B|} \int_{B}\left|\prod_{j=1}^{m}\left(b_{j}(y)-\left(b_{j}\right)_{B}\right) L^{-\alpha / 2} f(y)\right| d y \\
& \quad+\frac{1}{|B|} \int_{B}\left|\sum_{j=1}^{m-1} \sum_{\sigma \in C_{j}^{m}} C_{j, m}\left(b(y)-b_{B}\right)_{\sigma} L_{\vec{b}_{\sigma^{\prime}}}^{-\alpha / 2} f(y)\right| d y
\end{aligned}
$$

$$
\begin{aligned}
& +\frac{1}{|B|} \int_{B}\left|L^{-\alpha / 2}\left(\prod_{j=1}^{m}\left(b_{j}-\left(b_{j}\right)_{B}\right) f_{1}\right)(y)\right| d y \\
& +\frac{1}{|B|} \int_{B}\left|e^{-t_{B} L}\left(\prod_{j=1}^{m}\left(b_{j}-\left(b_{j}\right)_{B}\right) L^{-\alpha / 2} f\right)(y)\right| d y \\
& +\frac{1}{|B|} \int_{B}\left|\sum_{j=1}^{m-1} \sum_{\sigma \in C_{j}^{m}} C_{j, m} e^{-t_{B} L}\left(\left(b-b_{B}\right)_{\sigma} L_{\vec{b}_{\sigma^{\prime}}}^{-\alpha / 2} f\right)(y)\right| d y \\
& +\frac{1}{|B|} \int_{B}\left|e^{-t_{B} L}\left(L^{-\alpha / 2}\left(\prod_{j=1}^{m}\left(b_{j}-\left(b_{j}\right)_{B}\right) f_{1}\right)\right)(y)\right| d y \\
& +\frac{1}{|B|} \int_{B} \mid L^{-\alpha / 2}\left(\prod_{j=1}^{m}\left(b_{j}-\left(b_{j}\right)_{B}\right) f_{2}\right)(y) \\
& \quad-e^{-t_{B} L}\left(L^{-\alpha / 2}\left(\prod_{j=1}^{m}\left(b_{j}-\left(b_{j}\right)_{B}\right) f_{2}\right)\right)(y) \mid d y
\end{aligned}
$$$$
:=\sum_{i=1}^{7} G_{i}
$$

We now estimate the above seven terms, respectively. We take $m=2$ as an example; the estimate for the case $m>2$ is the same. For the first term $G_{1}$, we split it as follows:

$$
\begin{aligned}
G_{1}= & \frac{1}{|B|} \int_{B}\left|b_{1}(y)-\left(b_{1}\right)_{B, w}\right|\left|b_{2}(y)-\left(b_{2}\right)_{B, w}\right|\left|L^{-\alpha / 2} f(y)\right| d y \\
& +\frac{1}{|B|} \int_{B}\left|b_{1}(y)-\left(b_{1}\right)_{B, w} \|\left(b_{2}\right)_{B, w}-\left(b_{2}\right)_{B}\right|\left|L^{-\alpha / 2} f(y)\right| d y \\
& +\frac{1}{|B|} \int_{B}\left|\left(b_{1}\right)_{B, w}-\left(b_{1}\right)_{B} \| b_{2}(y)-\left(b_{2}\right)_{B, w}\right|\left|L^{-\alpha / 2} f(y)\right| d y \\
& +\frac{1}{|B|} \int_{B}\left|\left(b_{1}\right)_{B, w}-\left(b_{1}\right)_{B}\left\|\left(b_{2}\right)_{B, w}-\left(b_{2}\right)_{B}\right\| L^{-\alpha / 2} f(y)\right| d y \\
: & =G_{11}+G_{12}+G_{13}+G_{14} .
\end{aligned}
$$

Choose $r_{1}, r_{2}, r, q>1$ such that $1 / r_{1}+1 / r_{2}+1 / r+1 / q=1$. Noticing that $w \in A_{1}$, by Hölder's inequality and the similar estimate of $I_{1}$, we have

$$
G_{1} \leq C\|\vec{b}\|_{*} M_{r, w}\left(L^{-\alpha / 2} f\right)(x)
$$

For $G_{2}$, take $\tau_{1}, \ldots, \tau_{j}, \tau, v>1$ that satisfy $1 / \tau_{1}+\cdots+$ $1 / \tau_{j}+1 / \tau+1 / \nu=1$; then following Hölder's inequality and the same idea as that of $G_{1}$ yields

$$
G_{2} \leq C \sum_{j=1}^{m-1} \sum_{\sigma \in C_{j}^{m}} C_{j, m}\left\|\vec{b}_{\sigma}\right\|_{*} M_{\tau, w}\left(L_{\vec{b}_{\sigma^{\prime}}}^{-\alpha / 2} f\right)(x)
$$


To estimate $G_{3}$, applying Lemma 15 (Kolmogorov's inequality), weak $\left(1, n /(n-\alpha)\right.$ ) boundedness of $L^{-\alpha / 2}$ (see Remark 22), and Hölder's inequality, we have

$$
\begin{aligned}
G_{3} & =\frac{1}{|B|} \int_{B}\left|L^{-\alpha / 2}\left(\prod_{j=1}^{m}\left(b_{j}-\left(b_{j}\right)_{B}\right) f_{1}\right)(y)\right| d y \\
& \leq \frac{C}{|B|^{1-\alpha / n}} \| L^{-\alpha / 2}\left(\prod_{j=1}^{m}\left(b_{j}-\left(b_{j}\right)_{B}\right) f_{1}\right)||_{L^{n /(n-\alpha), \infty}} \\
& \leq \frac{C}{|B|^{1-\alpha / n}} \int_{2 B}\left|\prod_{j=1}^{m}\left(b_{j}(y)-\left(b_{j}\right)_{B}\right) f(y)\right| d y .
\end{aligned}
$$

We consider the case of $m=2$, for example, and we split $G_{3}$ as follows:

$$
\begin{aligned}
G_{3} \leq & \frac{C}{|B|^{1-\alpha / n}} \int_{2 B}\left(\left|b_{1}(y)-\left(b_{1}\right)_{2 B}\right|+\left|\left(b_{1}\right)_{2 B}-\left(b_{1}\right)_{B}\right|\right) \\
& \times\left(\left|b_{2}(y)-\left(b_{2}\right)_{2 B}\right|\right. \\
& \left.\quad+\left|\left(b_{2}\right)_{2 B}-\left(b_{2}\right)_{B}\right|\right)|f(y)| d y \\
\leq & \frac{C}{|B|^{1-\alpha / n}} \int_{2 B}\left|b_{1}(y)-\left(b_{1}\right)_{2 B}\right|\left|b_{2}(y)-\left(b_{2}\right)_{2 B}\right||f(y)| d y \\
& +\frac{C}{|B|^{1-\alpha / n}} \int_{2 B}\left|b_{1}(y)-\left(b_{1}\right)_{2 B}\right|\left|\left(b_{2}\right)_{2 B}-\left(b_{2}\right)_{B}\right||f(y)| d y \\
& +\frac{C}{|B|^{1-\alpha / n}} \int_{2 B}\left|\left(b_{1}\right)_{2 B}-\left(b_{1}\right)_{B}\right|\left|b_{2}(y)-\left(b_{2}\right)_{2 B}\right||f(y)| d y \\
& +\frac{C}{|B|^{1-\alpha / n}} \int_{2 B}\left|\left(b_{1}\right)_{2 B}-\left(b_{1}\right)_{B}\right|\left|\left(b_{2}\right)_{2 B}-\left(b_{2}\right)_{B}\right||f(y)| d y \\
:= & G_{31}+G_{32}+G_{33}+G_{34} .
\end{aligned}
$$

Take $r_{1}, r_{2}, r, q>1$ such that $1 / r_{1}+1 / r_{2}+1 / r+1 / q=1$; by virtue of Hölder's inequality and the same manner as that used in dealing with $I_{1}, I_{2}, I_{3}$ in Lemma 24, we get

$$
G_{31}+G_{32}+G_{33} \leq C\|\vec{b}\|_{*} M_{\alpha, r, w} f(x) .
$$

For $G_{34}$,

$$
\begin{aligned}
G_{34} & \leq C\|\vec{b}\|_{*} \frac{1}{|B|^{1-\alpha / n}} \int_{2 B}|f(y)| d y \\
& \leq C\|\vec{b}\|_{*} M_{\alpha, 1} f(x) .
\end{aligned}
$$

Therefore,

$$
G_{3} \leq C\|\vec{b}\|_{*} M_{\alpha, r, w} f(x)+C\|\vec{b}\|_{*} M_{\alpha, 1} f(x) .
$$

By Lemma 24, we have

$$
\begin{gathered}
G_{4} \leq C\|\vec{b}\|_{*} M_{r, w}\left(L^{-\alpha / 2} f\right)(x), \\
G_{5} \leq C \sum_{j=1}^{m-1} \sum_{\sigma \in C_{j}^{m}} C_{j, m}\left\|\vec{b}_{\sigma}\right\|_{*} M_{\tau, w}\left(L_{\vec{b}_{\sigma^{\prime}}}^{-\alpha / 2} f\right)(x) .
\end{gathered}
$$

Next, we consider the term $G_{6}$ :

$$
\begin{aligned}
G_{6}=\frac{1}{|B|} \int_{B} \mid \int_{\mathbb{R}^{n}} p_{t_{B}}(y, z) \\
\quad \times\left(L^{-\alpha / 2}\left(\prod_{j=1}^{m}\left(b_{j}-\left(b_{j}\right)_{B}\right) f_{1}\right)\right)(z) d z \mid d y \\
\leq \frac{1}{|B|} \int_{B} \int_{2 B}\left|p_{t_{B}}(y, z)\right| \\
\quad \times\left|L^{-\alpha / 2}\left(\prod_{j=1}^{m}\left(b_{j}-\left(b_{j}\right)_{B}\right) f_{1}\right)(z)\right| d z d y \\
\leq \frac{1}{|B|} \int_{B} \int_{\mathbb{R}^{n} \backslash 2 B}\left|p_{t_{B}}(y, z)\right| \\
\quad \times\left|L^{-\alpha / 2}\left(\prod_{j=1}^{m}\left(b_{j}-\left(b_{j}\right)_{B}\right) f_{1}\right)(z)\right| d z d y \\
:=G_{61}+G_{62} .
\end{aligned}
$$

For $G_{61}$, since $y \in B, z \in 2 B$, and $\left|p_{t_{B}}(y, z)\right| \leq C|2 B|^{-1}$, it follows that

$$
G_{61} \leq \frac{C}{|2 B|} \int_{2 B}\left|L^{-\alpha / 2}\left(\prod_{j=1}^{m}\left(b_{j}-\left(b_{j}\right)_{B}\right) f_{1}\right)(z)\right| d z .
$$

Analogous to the estimate of $G_{3}$, we have

$$
G_{61} \leq C\|\vec{b}\|_{*} M_{\alpha, r, w} f(x)+C\|\vec{b}\|_{*} M_{\alpha, 1} f(x) .
$$

For $G_{62}$, note that $y \in B, z \in 2^{k+1} B \backslash 2^{k} B$, and $\left|p_{t_{B}}(y, z)\right| \leq$ $C\left(e^{-C 2^{2(k-1)}} 2^{(k+1) n} /\left|2^{k+1} B\right|\right)$. Hence, the estimate of $G_{62}$ runs as that of $G_{61}$ yields that

$$
G_{62} \leq C\|\vec{b}\|_{*} M_{\alpha, r, w} f(x)+C\|\vec{b}\|_{*} M_{\alpha, 1} f(x) .
$$

For the last term $G_{7}$, applying Lemma 23, we have

$$
G_{7} \leq \frac{1}{|B|} \int_{B} \mid\left(L^{-\alpha / 2}-e^{-t_{B} L} L^{-\alpha / 2}\right)
$$

$$
\times\left(\prod_{j=1}^{m}\left(b_{j}-\left(b_{j}\right)_{B}\right) f_{2}\right)(y) \mid d y
$$

$$
\leq \frac{1}{|B|} \int_{B} \int_{\mathbb{R}^{n} \mid 2 B}\left|\widetilde{K}_{\alpha, t_{B}}(y, z)\right|
$$

$$
\times\left|\prod_{j=1}^{m}\left(b_{j}(z)-\left(b_{j}\right)_{B}\right) f(z)\right| d z d y
$$




$$
\begin{aligned}
& \leq C \sum_{k=1}^{\infty} \int_{2^{k+1} B \backslash 2^{k} B} \frac{t_{B}}{\left|x_{0}-z\right|^{n-\alpha+2}} \\
& \quad \times\left|\prod_{j=1}^{m}\left(b_{j}(z)-\left(b_{j}\right)_{B}\right) f(z)\right| d z \\
& \leq C \sum_{k=1}^{\infty} \frac{2^{-2 k}}{\left|2^{k} B\right|^{1-\alpha / n}} \int_{2^{k+1} B}\left|\prod_{j=1}^{m}\left(b_{j}(z)-\left(b_{j}\right)_{B}\right)\right||f(z)| d z .
\end{aligned}
$$

The same manner as that of $G_{3}$ gives us that

$$
\begin{aligned}
G_{7} & \leq C \sum_{k=1}^{\infty} 2^{-2 k}\left(\|\vec{b}\|_{*} M_{\alpha, r, w} f(x)+\|\vec{b}\|_{*} M_{\alpha, 1} f(x)\right) \\
& \leq C\|\vec{b}\|_{*}\left(M_{\alpha, r, w} f(x)+M_{\alpha, 1} f(x)\right) .
\end{aligned}
$$

According to the above estimates, we have completed the proof of Lemma 25.

\section{Proof of Theorems}

At first, we give the proof of Theorem 10.

Proof. It follows from Lemmas 13, 14, 25, and 17-21 that

$$
\begin{aligned}
& \left\|L_{\vec{b}}^{-\alpha / 2} f\right\|_{L^{q, \kappa q / p}\left(w^{q / p}, w\right)} \\
& \leq\left\|M L_{\vec{b}}^{-\alpha / 2} f\right\|_{L^{q, k q / p}\left(w^{q / p}, w\right)} \\
& \leq\left\|M_{L}^{\sharp}\left(L_{\vec{b}}^{-\alpha / 2} f\right)\right\|_{L^{q, \kappa q / p}\left(w^{q / p}, w\right)} \\
& \leq C\left\{\|\vec{b}\|_{*}\left\|M_{r, w}\left(L^{-\alpha / 2} f\right)\right\|_{L^{q, \kappa q / p}\left(w^{q / p}, w\right)}\right. \\
& +\sum_{j=1}^{m-1} \sum_{\sigma \in C_{j}^{m}} C_{j, m}\left\|\vec{b}_{\sigma}\right\|_{*} \\
& \times\left\|M_{\tau, w}\left(L_{\vec{b}_{\sigma^{\prime}}}^{-\alpha / 2} f\right)\right\|_{L^{q, \kappa q / p}\left(w^{q / p}, w\right)} \\
& +\|\vec{b}\|_{*}\left\|w^{-\alpha / n} M_{\alpha, r, w}(f)\right\|_{L^{q, k q / p}\left(w^{q / p}, w\right)} \\
& \left.+\|\vec{b}\|_{*}\left\|M_{\alpha, 1}(f)\right\|_{L^{q, \kappa q / p}\left(w^{q / p}, w\right)}\right\} \\
& \leq C\|\vec{b}\|_{*}\left\|L^{-\alpha / 2} f\right\|_{L^{q, \kappa q / p}\left(w^{q / p}, w\right)} \\
& +\sum_{j=1}^{m-1} \sum_{\sigma \in C_{j}^{m}} C_{j, m}\left\|\vec{b}_{\sigma}\right\|_{*}\left\|L_{\vec{b}_{\sigma^{\prime}}}^{-\alpha / 2} f\right\|_{L^{q, k q / p}\left(w^{q / p}, w\right)} \\
& +C\|\vec{b}\|_{*}\left\|M_{\alpha, r, w} f\right\|_{L^{q, \kappa q / p}(w)}+C\|\vec{b}\|_{*}\|f\|_{L^{p, \kappa}(w)} \\
& \leq C\|\vec{b}\|_{*}\|f\|_{L^{p, \kappa}(w)} \\
& +\sum_{j=1}^{m-1} \sum_{\sigma \in C_{j}^{m}} C_{j, m}\left\|\vec{b}_{\sigma}\right\|_{*}\left\|\left(L_{\vec{b}_{\sigma^{\prime}}}^{-\alpha / 2} f\right)\right\|_{L^{q, \kappa q / p}\left(w^{q / p}, w\right)} .
\end{aligned}
$$

Then, we can make use of an induction on $\sigma \subseteq\{1,2, \ldots, m\}$ to get that

$$
\left\|L_{\vec{b}}^{-\alpha / 2} f\right\|_{L^{q, \kappa q / p}\left(w^{q / p}, w\right)} \leq C\|\vec{b}\|_{*}\|f\|_{L^{p, \kappa}(w)} .
$$

This completes the proof of Theorem 10 .

Now, we are in the position of proving Theorem 11. If we take $\kappa=0$ in Theorem 10, we will immediately get our desired results.

\section{Conflict of Interests}

The authors declare that there is no conflict of interests regarding the publication of this paper.

\section{Acknowledgments}

The authors would like to express their gratitude to the anonymous referees for reading the paper carefully and giving some valuable comments. The research was partially supported by the National Nature Science Foundation of China under Grants nos. 11171306 and 11071065 and sponsored by the Scientific Project of Zhejiang Provincial Science Technology Department under Grant no. 2011C33012 and the Scientific Research Fund of Zhejiang Provincial Education Department under Grant no. Z201017584.

\section{References}

[1] X. T. Duong and L. X. Yan, "On commutators of fractional integrals," Proceedings of the American Mathematical Society, vol. 132, no. 12, pp. 3549-3557, 2004.

[2] S. Chanillo, "A note on commutators," Indiana University Mathematics Journal, vol. 31, no. 1, pp. 7-16, 1982.

[3] H. Mo and S. Lu, "Boundedness of multilinear commutators of generalized fractional integrals," Mathematische Nachrichten, vol. 281, no. 9, pp. 1328-1340, 2008.

[4] B. Muckenhoupt and R. L. Wheeden, "Weighted bounded mean oscillation and the Hilbert transform," Studia Mathematica, vol. 54, no. 3, pp. 221-237, 1976.

[5] H. Wang, "On some commutators theorems for fractional integral operators on the weighted Morrey spaces," http://arxivweb3.library.cornell.edu/abs/1010.2638v1.

[6] Z. Wang and Z. Si, "Commutator theorems for fractional integral operators on weighted Morrey spaces," Abstract and Applied Analysis, vol. 2014, Article ID 413716, 8 pages, 2014.

[7] Y. Komori and S. Shirai, "Weighted Morrey spaces and a singular integral operator," Mathematische Nachrichten, vol. 282, no. 2, pp. 219-231, 2009.

[8] B. Muckenhoupt, "Weighted norm inequalities for the Hardy maximal function," Transactions of the American Mathematical Society, vol. 165, pp. 207-226, 1972.

[9] J. García-Cuerva and J. L. R. de Francia, Weighted Norm Inequalities and Related Topics, North-Holland, Amsterdam, The Netherlands, 1985.

[10] J. M. Martell, "Sharp maximal functions associated with approximations of the identity in spaces of homogeneous type and applications," Studia Mathematica, vol. 161, no. 2, pp. 113-145, 2004 . 
[11] R. Johnson and C. J. Neugebauer, "Change of variable results for $A_{p}$ - and reverse Hölder $R_{H_{r}}$-classes," Transactions of the American Mathematical Society, vol. 328, no. 2, pp. 639-666, 1991.

[12] A. Torchinsky, Real-Variable Methods in Harmonic Analysis, Academic Press, San Diego, Calif, USA, 1986. 


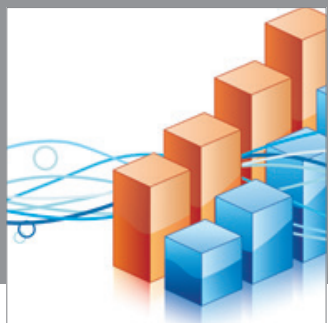

Advances in

Operations Research

mansans

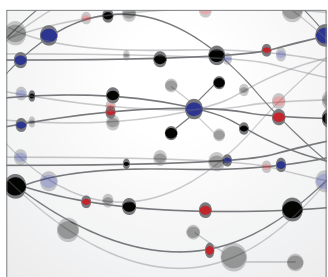

The Scientific World Journal
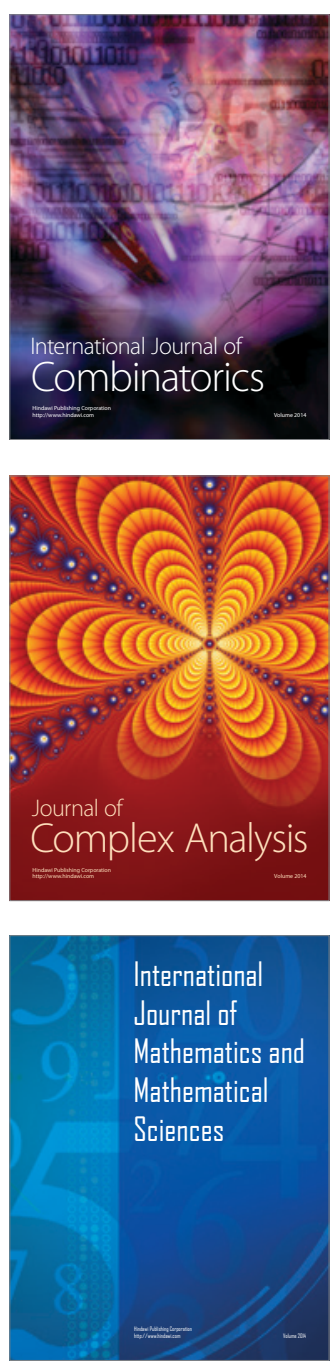
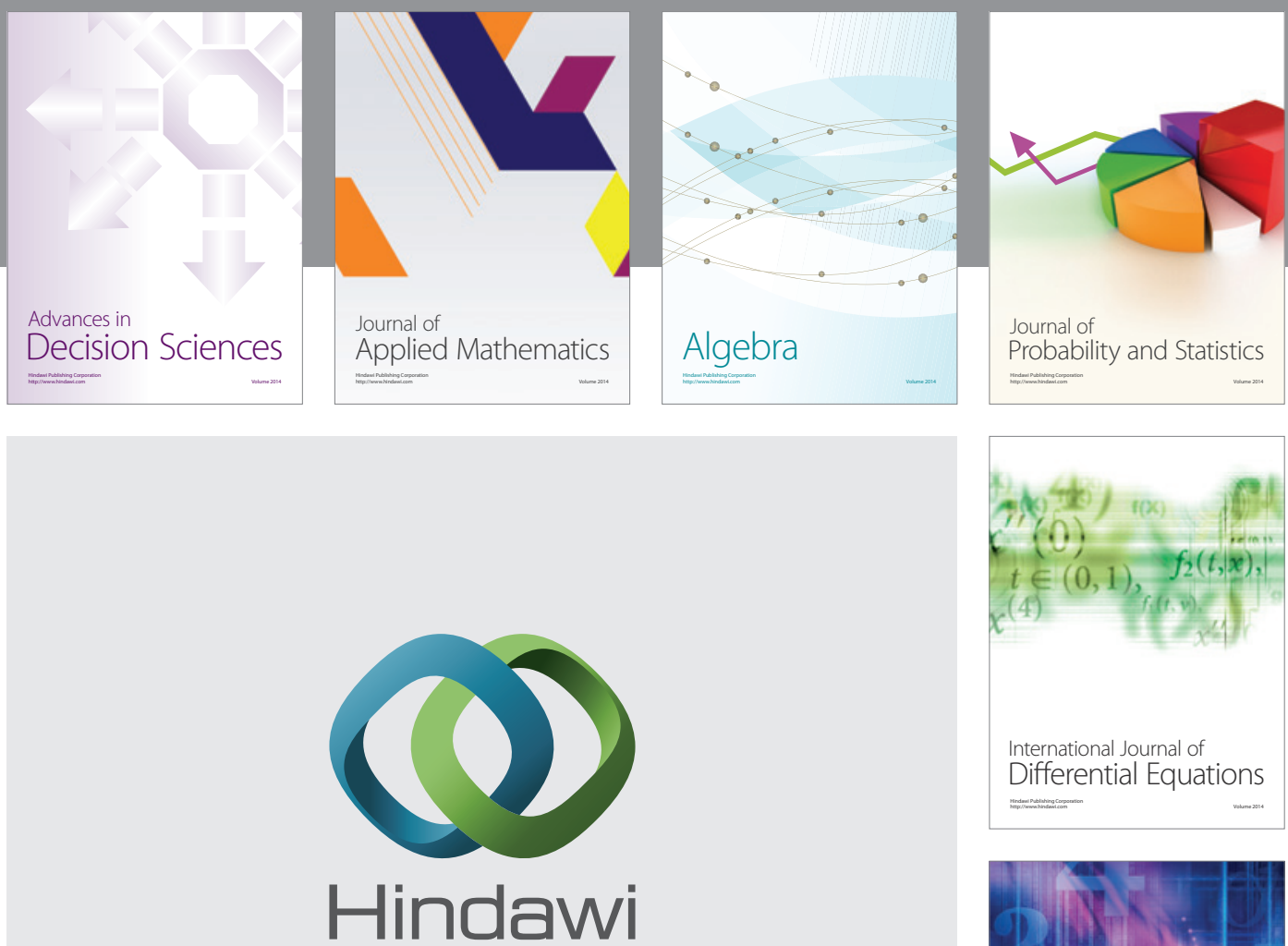

Submit your manuscripts at http://www.hindawi.com
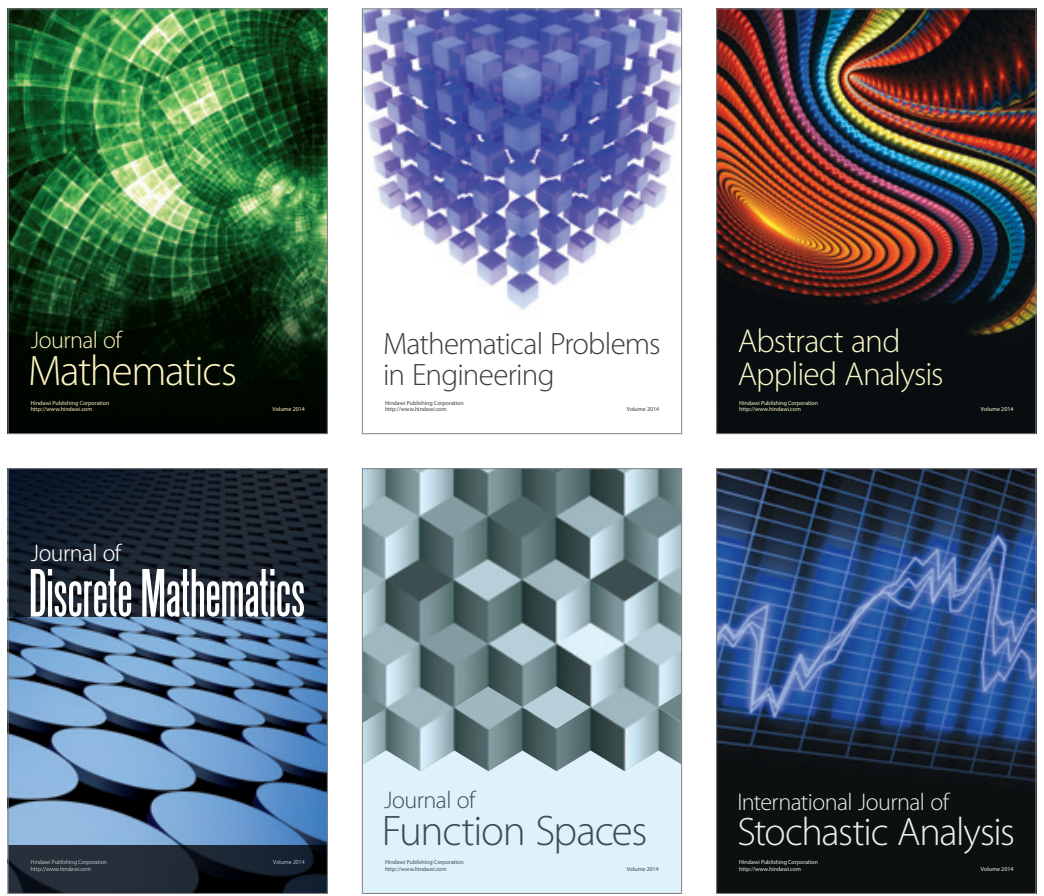

Journal of

Function Spaces

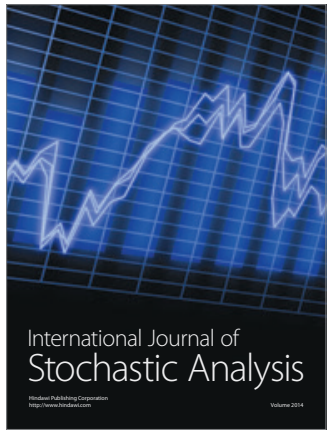

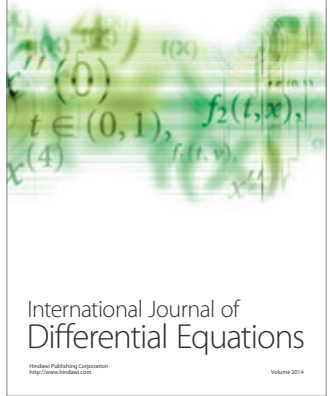
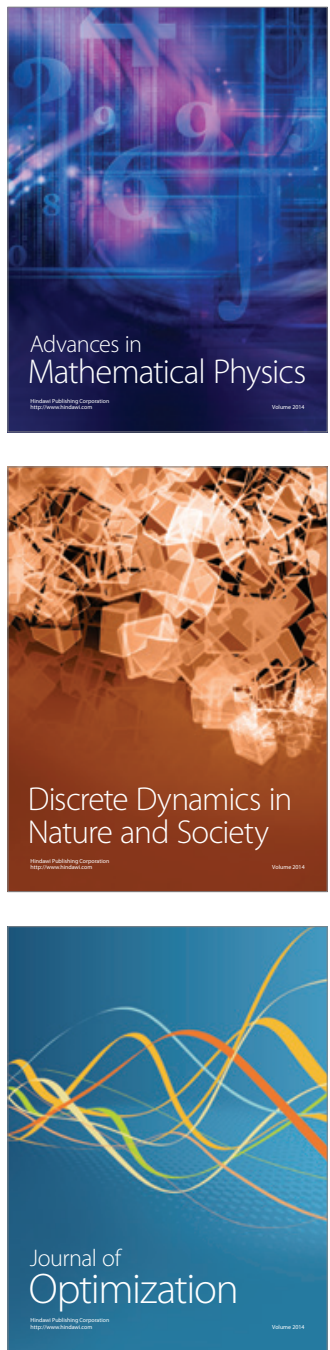